\title{
L'apprentissage de la palpation thérapeutique à partir de six procédures palpatoires haptiques : outils de formation et d'évaluation
}

\author{
Learning therapeutic palpation in six haptic palpatory procedures: training \\ and assessment tools.
}

\author{
Arnaud CHOPLIN ${ }^{1}$ \\ 1 Institut de Formation en Masso-Kinésithérapie Niçois, Centre Hospitalier Universitaire de l'Archet, \\ 151 Route Saint Antoine de Ginestière, 06202 Nice Cedex 3, France
}

Manuscrit reçu le 30 mai 2014 ; commentaires éditoriaux formulés aux auteurs le 20 novembre 2014 et le 7 mars 2015 ; accepté pour publication le 10 mars 2015

\author{
Mots-clés \\ Sensation haptique : \\ Perception ; \\ Apprentissage du \\ toucher; Toucher \\ thérapeutique ; \\ Palpation
}

\begin{abstract}
Résumé - Contexte et problématique : Le référentiel métier des masseurs-kinésithérapeutes précise des compétences palpatoires, véritable enjeu pour cette profession. C'est pourquoi la qualité de l'apprentissage palpatoire des étudiants en masso-kinésithérapie est fondamentale. Objectif : Vérifier l'hypothèse selon laquelle un dispositif pédagogique utilisant le modèle d'imitation-modélisation interactive (IMI) influence l'habileté gestuelle des étudiants de premier cycle. Méthodes : 124 étudiants de premier cycle d'études d'un institut de formation en masso-kinésithérapie ont été répartis égalitairement et de façon aléatoire en deux groupes. Durant les trois ans de l'étude (2010 à 2013), les étudiants de trois promotions ont bénéficié d'un nombre d'heures de formation identique. Seuls les étudiants du groupe expérimental ont bénéficié, dans ce laps de temps, de 57 heures d'un dispositif pédagogique par IMI. Une grille d'évaluation a été élaborée à l'aide des travaux sur l'haptisme. La reproductibilité entre deux évaluateurs est qualifiée de « bonne » en regard du coefficient de Cohen's Kappa. Résultats : Les mesures recueillies confirment l'hypothèse de départ ; le dispositif pédagogique proposé influence l'utilisation de cinq procédures palpatoires sur six. Seule la procédure de frottement latéral n'est pas confirmée. Conclusion : Le dispositif pédagogique par IMI permet d'acquérir une habileté et une cohérence palpatoires.
\end{abstract}




$\begin{array}{ll}\text { Keywords } & \text { Abstract - Background: The professional frames of physiotherapists specify palpatory } \\ \text { Haptic sensation; } & \text { skills. This is a real challenge for professionals and explains why it is crucial that physiother- } \\ \text { Perception; } & \text { apy students learn and benefit from top-quality palpatory training. Objective: To assess if the } \\ \text { Developing the sense } & \text { hypothesis demonstrated that an educational tool using the interactive modelling/imitation } \\ \text { of touch; Therapeutic } & \text { (IMI) model influences undergraduates' body movement abilities. Method: 124 undergradu- } \\ \text { touch; Palpation } & \text { ate students studying at the IFMK (institute of physiotherapy training) institute were split } \\ & \text { equally and randomly into two groups. During the three-year study (2010-2013), students } \\ \text { from three classes received the same amount of training time. Students of GE (experimental } & \text { group) group are the only ones that benefited from 57 hours of training with the IMI peda- } \\ \text { gogical tool. An evaluation chart was developed using works on haptics. Based on Cohen's } \\ \text { Kappa coefficient replicability between two evaluators is described as "good". The statistical } \\ \text { results are significant and confirm the initial hypothesis. The proposed educational tool has } \\ \text { an impact on the use of five palpatory procedures out of six. The lateral friction procedure is } \\ \text { the only one that was not confirmed. Conclusion: The IMI pedagogical tool helped acquire } \\ \text { palpatory skills and develop consistency. }\end{array}$

\section{Introduction}

En France, la formation initiale des masseurs-kinésithérapeutes se déroule en deux cycles d'études répartis sur trois années. L'admission des étudiants à cette formation s'opère majoritairement en fonction d'un classement à l'issue du concours de fin de première année commune aux études de santé (PACES), organisé dans les facultés de médecine. Le décret de compétences $^{[1]}$ et le programme d'études initiales de 1989 prescrivent comme objectif l'acquisition d'une compétence palpatoire et massothérapique au terme de ce premier cycle d'études masso-kinésithérapiques $^{[2]}$. Pourtant, la réalité de l'acquisition de ce savoir-faire fait débat chez les acteurs de cette formation. En effet, les formateurs ont le sentiment qu'à l'issue de ce cycle de formation, les étudiants présentent une déficience en capacité palpatoire. Par ailleurs, la littérature ne précise pas quels dispositifs pédagogiques sont susceptibles de favoriser l'apprentissage de cette habileté palpatoire.

\section{Contexte}

Dans le cadre d'une étude préalable, nous avons cherché à vérifier le bien-fondé du sentiment des formateurs quant à la non-appropriation de la compétence palpatoire thérapeutique nécessaire aux futurs masseurs-kinésithérapeutes (MK). Un questionnaire différentiel ayant pour objectif de vérifier les propos rapportés autour de leur réalisation palpatoire a été élaboré et administré à des étudiants de deuxième cycle lors des stages en institution de santé ${ }^{[3]}$. Ces étudiants avaient bénéficié, lors de leur premier cycle d'études, de séquences de formation sur la gestuelle thérapeutique, de manière à ce que ce que l'expérience de cette compétence ne constitue pas un biais méthodologique $^{[4]}$.

Les résultats de cette enquête, réalisée en 2009 et publiée en 2012, montrent que les étudiants de deuxième cycle d'études en masso-kinésithérapie présentent une déficience palpatoire en ce qui concerne le démarrage d'une palpation thérapeutique et l'identification d'un élément anatomique précis et profond, telle que la mise en évidence du muscle piriforme ${ }^{[5]}$. En toute logique, ces étudiants devraient pourtant être compétents en regard du référentiel professionnel des masseurs-kinésithérapeutes ${ }^{[1]}$. Or nous constatons que ce n'est pas le cas. Notons que les textes législatifs qui encadrent les études de masso-kinésithérapie permettent aux étudiants de valider les modules par l'obtention de la moyenne. Ainsi paradoxalement, la validation du module, sur la base de l'obtention d'un score égal ou supérieur à la moyenne lors des épreuves telles qu'elles sont administrées, ne garantit pas que les étudiants aient acquis toutes les compétences d'habiletés gestuelles attendues. 


\section{Cadre conceptuel}

Une revue de la littérature issue du champ des neurosciences depuis les travaux de Revesz en 1934) ${ }^{[6]}$, de Gibson en $1966^{[7]}$ et, plus récemment, de Lederman en 1987 , de Klatzky en $1993^{[8,9]}$ et d'Hatwell en $2000^{[10]}$ établit qu'il existe six procédures palpatoires haptiques dans la reconnaissance tactile d'objets inconnus. La démarche semble suivre une stratégie immuable, en six temps, dès lors qu'on veut reconnaître un objet par l'utilisation du sens haptique (Annexe $n^{\circ} 1$ ). L'analogie avec le diagnostic palpatoire clinique semble séduisante.

Dès l'âge de cinq à six ans, lors d'une reconnaissance palpatoire, l'enfant procède manuellement et en première intention par une manœuvre «d'enveloppement global », qui lui permet de reconnaître rapidement la forme d'un objet. Les travaux de recherche résumés par Gentaz en 2009 permettent d'affirmer que les formes en relief sont mieux perçues et mieux discriminées que les formes en creux ${ }^{[11]}$. Dans l'hypothèse où l'individu n'a pas été en mesure de reconnaître l'objet palpé, il met en place en deuxième intention une stratégie de « suivi des contours » ${ }^{[11]}$. Dès lors, l'individu utilise une stratégie de perception haptique qui résulte de la stimulation de la peau à l'aide de mouvements actifs d'exploration ${ }^{[11]}$. Ce sens haptique est très performant pour percevoir la qualité de texture et de dureté des matériaux. Les travaux de Lederman ${ }^{[9]}$ et Klatzky ${ }^{[10]}$, repris par Hatwell ${ }^{[8]}$ puis par Gentaz ${ }^{[11]}$, permettent d'identifier six principales procédures exploratoires indispensables à l'identification d'un objet touché. Pour percevoir un élément par le toucher, le palpeur démarre par un enveloppement de l'objet, ce qui lui en donne une idée approximative. Puis il organise sa motricité par un suivi des contours, ce qui lui permet de confirmer la forme exacte de l'objet. Le contact statique permet une indication de température et complète l'information sur la forme et la taille. Le frottement latéral permet de déterminer la texture, la pression permet de déterminer la dureté de l'objet. Le soupèsement détermine le poids de l'objet. En annexe $\mathrm{n}^{\circ} 1$, nous proposons une schématisation de ces six procédures adaptée à la pratique palpatoire thérapeutique de la structure osseuse du massif du grand trochanter à la face externe de hanche.

La palpation, qu'elle relève d'une dimension diagnostique par le médecin ou d'une application thérapeutique par le soignant, convoque inconsciemment ces six procédures haptiques en fonction de la tâche que le professionnel de santé a à réaliser. Klatzky et Gentaz montrent que le processus haptique est très performant, autant que la vision, pour l'identification d'un objet usuel connu ${ }^{[10-11]}$. La pratique palpatoire thérapeutique s'exerce donc comme une interprétation perceptive autour de cette habileté gestuelle en six temps.

Dans le cadre du dispositif de formation envisagé, il ne s'agit pas d'exiger que l'étudiant reproduise ces six procédures haptiques dans une suite logique, mais bien de proposer d'une part, un outil didactique au service du formateur et d'autre part, un cadre de référence au service de l'évaluation. De fait, nous ne nous inscrivons pas dans un dispositif pédagogique de type imitation à l'identique (mimétisme). Au contraire, nous souhaitons permettre aux étudiants d'utiliser ces procédures « à bon escient », d'apprendre à les utiliser en fonction des situations palpatoires recherchées. Cette dimension interprétative sensitive impose un dispositif pédagogique de type socioconstructiviste. Il s'agit de procéder à ce qui s'apparente à une «transposition didactique » au sens où l'envisage Chevallard ${ }^{[12]}$. Dans cette posture, le formateur utilise cet apport dans une dimension imitative à partir d'une situation montrée (modèle), dans une situation d'imitation-modélisation interactive au sens où $l^{\prime}$ 'entend Winnykamen ${ }^{[13]}$. Les six procédures constituent d'abord un outil de relation au sein de la dyade symétrique étudiant-étudiant et de la dyade asymétrique étudiant-formateur, dans un espace que Vygotski nomme zone proximale de développement $(\mathrm{ZPD})^{[14]}$. Elles représentent également, pour le formateur, un outil au service de la dimension évaluative. Utilisées sans être instruites directement, elles permettent à l'évaluateur d'appréhender voire de quantifier l'habileté gestuelle. Elles permettent ainsi une lisibilité de ce que perçoit l'apprenti palpeur. 


\section{Présentation des deux dispositifs pédagogiques}

Cette étude compare deux dispositifs pédagogiques différents dans une approche transmissive d'un geste palpatoire thérapeutique. Les deux dispositifs peuvent être présentés en trois phases autour d'une démonstration réalisée par le formateur et que l'étudiant devra «imiter » selon deux modalités distinctes :

- imitation mimétique à l'identique pour les étudiants du groupe témoin (GT) ;

- imitation-modélisation interactive (IMI) pour les étudiants du groupe expérimental (GE).

Dans le cadre du dispositif qui applique une logique pédagogique qualifiée d' «habituelle», auquel sont exposés les étudiants du GT :

- la séquence pédagogique de pré-démonstration repose sur une présentation anatomique préalable. Cet espace théorique doit permettre à l'étudiant de faire émerger des représentations de ce qu'il devra réaliser pour réussir la prescription palpatoire. De cette théorie, des habiletés gestuelles émergent. Des règles de «bonne » pratique semblent incontournables. Le formateur attire l'attention sur des invariants palpatoires, afin de favoriser la projection de ce que l'étudiant devra réaliser.

- Puis le formateur réalise une démonstration devant l'ensemble des étudiants du GT. Durant cette séquence pédagogique, les étudiants interrogent le formateur de façon exhaustive. L'intérêt pour eux est de s'approprier un maximum de détails afin de pouvoir reproduire l'acte palpatoire en reproduisant le plus exactement ce qu'ils ont vu. Cette démonstration est réalisée lentement, séquencée et répétée autant de fois que nécessaire. Le formateur répond aux questions et donne des consignes précises.

- En post-démonstration, le formateur contrôle les gestes palpatoires sans laisser de possibilités créatives ou innovantes aux étudiants. Le formateur corrige les habiletés gestuelles en conformité avec ce qu'il a préalablement montré.

L'étudiant doit assimiler puis reproduire la palpation modèle, en l'accommodant conformément à la démonstration ${ }^{[15]}$.
Dans le cadre du dispositif qui applique une logique pédagogique qualifiée de «spécifique», auquel sont exposés les étudiants du GE :

- lors de la séquence pédagogique de pré-démonstration, le formateur, par un jeu de questions, provoque des interactions entre les étudiants relativement à la tâche palpatoire prescrite. Des idées, des opinions s'affrontent. Des conflits naissent. Le formateur met en place les conditions de l'émergence des représentations palpatoires préalables, en amenant les étudiants à imaginer des processus réalisables, ce qui favorise l'anticipation par les étudiants de ce qu'ils auront à réaliser ${ }^{[16]}$.

- Puis le formateur effectue une démonstration devant l'ensemble des étudiants du GE. Durant cette séquence pédagogique, les étudiants n'interrogent pas le formateur sur ce qu'il est en train de faire. La démonstration-modèle s'exécute à vitesse réelle, identique à ce qui se pratique dans un cadre professionnel. Le formateur souligne la possibilité d'une ouverture sur d'autres techniques possibles. À ce niveau, la nature de la démonstration oblige l'étudiant à une imitation du modèle et non à une imitation du formateur. L'étudiant est dans l'incapacité de reproduire les gestes à l'identique. Il doit mobiliser les représentations élaborées précédemment.

- En post-démonstration, lorsque l'étudiant doit à son tour réaliser l'acte palpatoire, il n'a d'autres choix que de créer et innover. L'étudiant montre, formalise ce qu'il serait possible de faire pour répondre à la prescription palpatoire. Il élabore ainsi son style palpatoire en conformité avec le genre métier ${ }^{[16]}$. Le formateur questionne l'étudiant sur ce qu'il est en train de faire. Ces questions sont centrées implicitement sur l'utilisation des six procédures palpatoires haptiques. Ainsi, le formateur amène l'étudiant à s'approprier les procédures haptiques dans une zone proximale de développement ${ }^{[13]}$. Il accompagne l'étudiant dans une stratégie de guidage-tutelle, en proposant une imitation modélisation-interactive ${ }^{[13,18]}$. L'étudiant s'approprie l'utilisation de ces six procédures haptiques dans une optimisation perceptive observable par les habiletés gestuelles. 
Tableau I. Présentation du tri à plat des collectifs de l'étude.

\begin{tabular}{|l|c|c|c|c|c|c|c|c|c|}
\hline \multirow{2}{*}{ Nbre total = 124 } & \multicolumn{3}{|c|}{ Promotion 2010-2011 } & \multicolumn{2}{c|}{ Promotion 2011-2012 } & \multicolumn{3}{c|}{ Promotion 2012-2013 } \\
\cline { 2 - 10 } & Nbre total & GT & GE & Nbre total & GT & GE & Nbre total & GT & GE \\
\hline effectif & 42 & 20 & 22 & 42 & 21 & 21 & 40 & 20 & 20 \\
\hline Ratio sexe & 1,6 & 1,8 & 1,4 & 1,2 & 1,2 & 1,2 & 1,2 & 1,5 & 1 \\
\hline Moyenne âge & 20,5 & 19,3 & 20,7 & 20 & 19,8 & 20,2 & 19,5 & 18,8 & 18,6 \\
\hline Ecart type & 1,3 & 0,73 & 1,87 & 0,75 & 0,76 & 0,92 & 0,65 & 0,64 & 0,64 \\
\hline Mode & 20 & 20 & 19 & 20 & 20 & 20 & 20 & 20 & 19 \\
\hline médiane & 20 & 20 & 20 & 20 & 20 & 20 & 19,5 & 20 & 19 \\
\hline
\end{tabular}

GT : groupe témoin : GE : groupe expérimental

\section{Objectif}

L'objectif de ce travail est de vérifier l'hypothèse selon laquelle un dispositif pédagogique utilisant le modèle d'imitation-modélisation interactive (IMI) ${ }^{[12]}$ influence l'habileté gestuelle des étudiants de premier cycle. Plus spécifiquement, nous cherchons à montrer que les étudiants de premier cycle d'études en masso-kinésithérapie ayant bénéficié d'un dispositif pédagogique de type "spécifique ${ }^{[12]}$ utilisent de façon plus optimale les six procédures palpatoires haptiques décrites dans le cadre de l'approche neuroscientifique de Lederman et Klatzky ${ }^{[9-10]}$.

\section{Méthodes}

\section{Conditions du déroulement de l'étude}

Le dispositif pédagogique d'imitation-modélisation interactive administré aux étudiants du groupe expérimental (GE) se déroule en 57 heures de cours, qui s'inscrivent dans le cadre d'un dispositif de 120 heures pendant les 19 semaines de l'étude. Dans le même laps de temps, les étudiants du groupe témoin (GT) bénéficient d'un enseignement « habituel », tel qu'il a été exposé.

Protocole et tri à plat

L'étude expérimentale randomisée, monocentrique, d'une durée de trois ans (de 2010 à 2013) a été conduite dans un institut de formation en massokinésithérapie (IFMK). Cent vingt-quatre étudiants de premier cycle d'études de masso-kinésithérapie ont été répartis aléatoirement en deux groupes, 61 étudiants pour le GT et 63 étudiants pour le GE. Cet échantillon est représentatif de la population des étudiants en masso-kinésithérapie de France. Le sexe-ratio de cet échantillon est de 1,3 en faveur des filles, la moyenne d'âge, le mode et la médiane sont de 20 pour un écart type de 0,5 (tableau I). Ces premiers résultats du tri à plat nous permettent d'affirmer que notre cohorte est homogène.

Le parcours scolaire de notre échantillon a comporté, pour plus de $98 \%$ d'entre eux, un baccalauréat (diplôme de fin d'études secondaires en France) scientifique (série $\mathrm{S}$ ) ou économique et social (série ES), suivi de la PACES. Ceci signifie que les étudiants de l'étude ont été admis en premier cycle d'études de masso-kinésithérapie après un concours classant. Cette étude s'est déroulée chaque année de mi-octobre à mi-mars, soit durant 19 semaines consécutives. Sur les 120 heures de formation dite habituelle, le groupe expérimental a bénéficié de 57 heures de pédagogie dite «spécifique », telle qu'elle est précédemment décrite.

\section{Modalités de réalisation et d'évaluation}

Nous avons mis en place un dispositif d'évaluationmesure des performances observées, afin de mesurer l'impact de ces deux dispositifs pédagogiques sur l'appropriation d'une compétence palpatoire 
Tableau II. Répartition des dates d'évaluations initiale et finale de l'étude.

\begin{tabular}{|c|c|c|}
\hline & $\begin{array}{c}\text { Dates évaluation } \\
\text { initiale }\end{array}$ & $\begin{array}{c}\text { Dates évaluation } \\
\text { finale }\end{array}$ \\
\hline $2010-2011$ & 14 octobre 2010 & 07 mars 2011 \\
\hline $2011-2012$ & 21 octobre 2011 & 13 mars 2012 \\
\hline $2012-2013$ & 09 octobre 2012 & 12 mars 2013 \\
\hline
\end{tabular}

thérapeutique. Le tableau II présente les dates des évaluations initiales et finales :

Toutes les palpations ont été réalisées sur un camarade de promotion comme c'est la coutume dans les IFMK. Dans des conditions identiques, tous les étudiants ont une région anatomique à palper sans aucune consigne particulière.

Lors de ces évaluations, la variable dépendante «qualité palpatoire » est évaluée à partir de ce que l'apprenti palpeur réalise comme habiletés gestuelles en regard des six procédures palpatoires. Notre grille d'évaluation, présentée en Annexe $n^{\circ}$ 2, est qualifiée de binaire: elle vérifie si l'habileté gestuelle, en regard des six procédures haptiques, a été réalisée ou non. Cette évaluation-contrôle traduit donc l'utilisation du nombre de procédures palpatoires utilisées lors d'une réalisation palpatoire prescrite ${ }^{[18]}$. Rappelons que dans aucun des deux dispositifs pédagogiques les étudiants ne sont informés explicitement de l'existence de ce modèle procédural haptique en six procédures. Dans ces conditions, le nombre de procédures utilisé traduit l'habileté gestuelle du palpeur à mettre en évidence un élément anatomique. Il s'agit de quantifier la qualité palpatoire, c'est à dire l'habileté gestuelle des étudiants palpeurs en référence à un modèle théorique issu des neurosciences.

L'étudiant doit réaliser sa prescription palpatoire sans obligation d'expliquer oralement ce qu'il est en train de faire, s'il s'agit pour lui d'une difficulté supplémentaire. Le temps de palpation a été laissé volontairement libre. En février-mars, les étudiants ont été interrogés dans les mêmes conditions et évalués suivant des critères et indicateurs identiques. Le recueil des données mesurant l'habileté gestuelle des étudiants a été réalisé à l'aide d'un outil créé pour la circonstance $\left(\right.$ Annexe $\left.\mathrm{n}^{\circ} 2\right)$.
La grille d'évaluation est utilisée selon les mêmes modalités entre les dix-neuf semaines qui séparent l'évaluation initiale et l'évaluation finale. Un point est attribué par procédure palpatoire réalisée, soit un score maximal de six points, lorsque l'évaluateur observe un des six indicateurs palpatoires décrits par les travaux de Lederman ${ }^{[9]}$. Cette cotation binaire (0 ou 1$)$ rend compte du geste pratique mis en oeuvre par l'étudiant lors de sa réalisation palpatoire.

Les 124 étudiants du premier cycle de l'IFMK participant à l'étude ont donc été évalués deux fois (évaluation initiale et finale), dans les locaux de l'institut, par une élève de Master de l'Université d'AixMarseille 1, suivant les critères et indicateurs préétablis à l'aide de la grille d'évaluation (Annexe $\left.\mathrm{n}^{\circ} 2\right)$.

\section{Vérification préalable du caractère reproductible de la mesure à l'aide de la grille d'évaluation}

La grille d'évaluation, créée pour la circonstance, a fait l'objet d'une vérification métrologique préalable à son utilisation dans le cadre des évaluations de l'étude (en 2010), de manière à apprécier le pourcentage d'erreurs maximales tolérées (EMT). Avant toute investigation évaluative, nous avons mesuré la concordance des jugements de nature qualitative entre deux examinateurs : l'un cadre en massokinésithérapie et l'autre détenteur d'un Master en sciences de l'éducation. Douze étudiants de deuxième cycle d'études de masso-kinésithérapie, ne participant pas à l'étude, ont été sollicités pour réaliser des palpations en conformité avec celles prescrites aux étudiants de l'étude.

Nous avons ainsi testé notre grille d'évaluation et calculé le coefficient de Cohen's Kappa inter-examinateurs. L'Annexe $n^{\circ} 3$ rend compte de l'attribution des scores obtenus par les deux examinateurs lors des prestations palpatoires réalisées par les 12 étudiants de deuxième cycle d'études de masso-kinésithérapique du même IFMK.

Le tableau III présente la concordance entre les deux examinateurs de l'étude. D'après Landis ${ }^{[19]}$, le degré d'accord en fonction de la valeur de Kappa est qualifié de "bon " car le $k$ du coefficient de Cohen' Kappa est de 0,67, compris entre 0,61 
Tableau III. Concordances entre les deux examinateurs lors de l'usage de la grille d'èvaluation.

\begin{tabular}{|l|l|l|}
\hline Po & 0,7500 & Pourcentage brut d'accords \\
\hline Pe & 0,2431 & Facteur de correction \\
\hline K & 0,6697 & Valeur corrigée = Kappa \\
\hline ET $k$-n & 0,1546 & Erreur type du Kappa \\
\hline I.C. [ & 0,3666 & Borne inférieure de l'intervalle de confiance \\
\hline I.C. $]$ & 0,9728 & Borne supérieure de l'intervalle de confiance \\
\hline$Z$ & 4,3311 & Cote $Z$ \\
\hline P & 0,0000 & Probabilité \\
\hline Donc & $P<0,05$ & Conclusion statistique \\
\hline
\end{tabular}

Tableau IV. Nombre de procédures haptiques utilisées dans les groupes témoin et expérimental lors de l'évaluation finale (post test).

\begin{tabular}{|l|c|c|c|}
\hline \multicolumn{1}{|c|}{ Les six procédures palpatoires } & Groupe Témoin & Groupe Expérimental & Différentiel \\
\hline Enveloppement & 9 & 44 & 35 \\
\hline Suivi des contours & 12 & 43 & 31 \\
\hline Contact statique & 1 & 15 & 6 \\
\hline Frottement latéral & 26 & 32 & 30 \\
\hline Pression-dépression & 11 & 41 & 9 \\
\hline Soupèsement & 1 & 10 & 14 \\
\hline
\end{tabular}

(limite inférieure) et 0,80 (limite supérieure), ce qui signifie que les deux examinateurs ont évalué l'habileté gestuelle de manière statistiquement identique à partir des données de la grille d'évaluation proposée (Annexe $\mathrm{n}^{\circ} 3$ ).

\section{Résultats}

L'annexe $n^{\circ} 4$ présente les données exhaustives brutes pour le groupe témoin et pour le groupe expérimental. Elle expose le nombre de procédures haptiques utilisées lors des évaluations initiale et finale pour chaque année d'étude.

Le tableau IV et la figure 1 montrent de façon synthétique que les habiletés gestuelles de démarrage palpatoire (enveloppement et suivi des contours) et d'identification d'un élément anatomique précis et profond (pression-dépression) sont utilisées quatre fois plus par les étudiants du GE que par ceux du GT en post-formation. Ce n'est pas le cas de la mise en évidence de structures anatomiques superficielles puisque, même si les étudiants du GE utilisent en postformation la procédure de frottement latéral 32 fois plus qu'en pré-formation contre 26 fois en ce qui concerne les étudiants du GT, la différence de 6 unités reste modeste. La recherche de la température (contact statique) et de la masse du membre palpé (soupèsement) s'améliore dans un ratio de 10 fois plus en faveur des étudiants du GE. Il s'agit de deux procédures utilisées dans une stratégie diagnostique.

En première analyse nous pensons que les situations dyadiques peuvent expliquer que les étudiants du GE utilisent de façon massive les six procédures haptiques.

Le calcul statistique du t de Student à l'aide du logiciel Excel permet de confirmer les résultats précédemment évoqués. Alors que la moyenne d'utilisation des six procédures palpatoires haptiques par les étudiants du GT $(1,4 / 6)$ est supérieure à celle du GE 


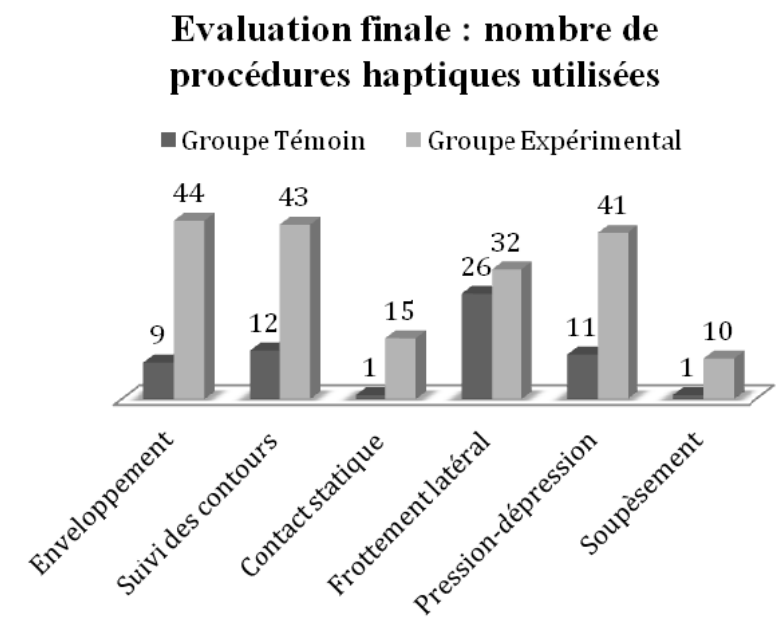

Fig. 1. Nombre de procédures haptiques utilisées respectivement par les étudiants du groupe témoin (GT) et du groupe expérimental (GE) lors de l'évaluation finale.

Tableau V. Moyennes du nombre de procédures palpatoires mises en œuvre par les étudiants du groupe expérimental (GE) et du groupe témoin (GT) lors des évaluations initiale et finale.

\begin{tabular}{|l|c|c|c|c|}
\cline { 2 - 5 } \multicolumn{1}{c|}{} & \multicolumn{4}{c|}{ Moyennes d'utilisation des six procédures palpatoires } \\
\cline { 2 - 5 } \multicolumn{1}{c|}{} & \multicolumn{2}{c|}{ Evaluation initiale (pré-test) } & \multicolumn{2}{c|}{ Evaluation finale (post-test) } \\
\cline { 2 - 5 } \multicolumn{1}{c|}{} & GE & GT & GE & GT \\
\hline moyennes & 0,9 & 1,4 & 4,2 & 0,9 \\
\hline écart type & 0,7 & 0,6 & 0,6 & \multicolumn{2}{c|}{$8,00 \mathrm{E}-10$} \\
\hline Test te Student & \multicolumn{2}{|c|}{$0,01(1 \%)$} & \multicolumn{2}{c|}{} \\
\hline
\end{tabular}

$(0,9 / 6)$ lors de l'évaluation initiale, l'inverse est constaté lors de l'évaluation finale (2,9/6 pour le GT vs. 4,2/6 pour le GE). Lors des deux évaluations (initiale et finale), les moyennes sont statistiquement différentes. Lors de l'évaluation initiale, c'est la moyenne du GT qui est la plus élevée, tandis que lors de l'évaluation finale c'est la moyenne du GE qui devient plus importante pour un risque $p$ très faible $\left(8^{\mathrm{E}}-10\right)$.

L'analyse statistique, procédure haptique par procédure haptique, lié à notre premier objectif montrent, dans les conditions de cette étude, que le dispositif de formation "spécifique » organisé à l'aide d'une «transposition didactique » permet aux étudiants du GE d'utiliser cinq procédures palpatoires haptiques sur les six, de façon statistiquement significative par rapport aux étudiants du $\mathrm{GT}^{[12,13]}$. Seule la progression de la procédure haptique de frottement latéral ne semble pas influencée par le type de formation proposée (tableau VI).

\section{Discussion}

Les résultats et l'analyse montrent que le dispositif pédagogique «spécifique » de type imitation-modélisation interactive défendu par Winnykamen ${ }^{[13]}$, mis en place en lien avec un modèle de type « transposition didactique $»^{[12]}$ influence de façon statistiquement significative la palpation thérapeutique haptique des étudiants de premier cycle d'études de massokinésithérapie. Avec ce dispositif pédagogique, l'enveloppement global et le suivi des contours sont 
utilisés quatre fois plus par les étudiants du groupe expérimental (GE) que par ceux du groupe témoin (GT) qui ont eu une formation « habituelle » basée sur des cohérences théorico-pratiques (anatomiques et biomécaniques). Ce résultat permet d'envisager une efficience quatre fois plus importante lors d'un dispositif pédagogique expérimental que lors d'une pédagogie habituelle (GT). C'est aussi le cas de la palpation d'un élément anatomique précis et profond comme le muscle piriforme. L'évaluation tactile de la température cutanée par le contact statique et l'évaluation de la masse par l'action de soupèsement sont effectuées significativement plus souvent dans le cadre du dispositif pédagogique expérimental.

Ces résultats positifs en faveur d'une pédagogie imitative d'inspiration socioconstructiviste permettent dans un premier temps d'outiller le formateur afin que les réalisations palpatoires thérapeutiques des étudiants être effectuées avec une habileté gestuelle plus performante.

Le partage de cet apport avec l'ensemble de la communauté des formateurs en charge de la transmission d'un geste palpatoire sur l'individu permet non seulement de proposer un dispositif performant mais aussi, dans un deuxième temps, de permettre, aidé d'un outil d'évaluation, de mesurer la qualité palpatoire de l'étudiant lors de la validation de son module. Il s'agit là de rendre plus efficient le système de contrôle des habiletés gestuelles avant que l'étudiant intègre un deuxième cycle d'études en masso-kinésithérapie.

Cependant, le caractère monocentrique de cette étude constitue une faiblesse qui doit conduire à examiner les conclusions de façon critique. Il aurait été judicieux d'évaluer ce dispositif expérimental dans le cadre d'une étude sollicitant plusieurs IFMK et un plus grand nombre d'étudiants. D'autre part, nous réduisons la capacité palpatoire haptique à six procédures, alors que le processus d'action palpatoire des étudiants est certainement plus complexe et qu'il faut probablement envisager des outils d'exploration plus pertinents. Nous pensons à l'utilisation de méthodes de recherche indirecte comme l'instruction au sosie ${ }^{[20]}$ ou encore l'autoconfrontation ${ }^{[21]}$, qui permettraient sans doute de comprendre le processus d'action de chaque étudiant par une discussion sur le «pourquoi », à partir de ce qu'il vient de faire.
Nous avons fait le choix d'une méthode quantitative de type expérimental, ce qui n'est pas sans poser de problèmes.

- Lors de l'évaluation initiale, cette étude a été présentée à l'ensemble des promotions, ce qui a provoqué une période d'interrogation et de stratégie d'étudiants pour connaître leur groupe d'appartenance. Ce «flottement»a été identifié par les étudiants comme une injustice pédagogique. Pour certains, les étudiants d'un groupe sont formés par la «bonne méthode » tandis que les autres bénéficient d'une méthode de moindre qualité. La qualité de l'annonce s'est donc améliorée de la première promotion à la troisième.

- Lors du déroulement et durant les trois années de l'étude, nous sommes conscients que les formateurs ont modifié leurs enseignements. Un biais apparait du fait même de la durée de cette étude. Nous nous sommes retrouvé face à un dilemme entre le désir d'augmenter le nombre d'étudiants inclus dans l'étude (dans des conditions les plus homogènes possibles) et les éventuels biais de transformation des compétences des formateurs. Nous ne sommes pas en mesure, non plus, de garantir le respect de la consigne qui était de ne pas échanger les cours entre étudiants des deux groupes. Cette relation inter-individus n'a pas été révélée mais nous ne pouvons garantir qu'elle n'ait pas eu lieu; d'une façon plus générale, nous ne pouvons certifier qu'il n'y a pas eu d'échange entre les étudiants des deux groupes.

Nous pouvons aussi facilement comprendre que le degré d'implication et le «stress » occasionné lors des évaluations initiale et finale constituent un biais. Toutefois, nous pensons qu'il s'agit d'une donnée identique entre les deux groupes de l'étude.

\section{Conclusion}

Ce travail accrédite la thèse selon laquelle un dispositif pédagogique utilisant une stratégie socioconstructiviste, mise en œuvre en lien avec un modèle d'imitation-modélisation interactive apporte une cohérence dans l'apprentissage des capacités palpatoires. L'émergence du lien entre l'habileté gestuelle 
Tableau VI. Comparaison du recours aux différentes procédures (nombre de fois) par les étudiants du groupe expérimental (GE) et du groupe témoin (GT), lors de l'évaluation initiale (pré-test) et de l'évalauation finale (post-test).

\begin{tabular}{|c|c|c|c|c|}
\hline \multirow{2}{*}{$n=124$} & \multicolumn{4}{|c|}{ Procédure d' enveloppement } \\
\hline & GE pré-test & GT pré-test & GE post-test & GT-post-test \\
\hline Moyenne/20 & 1,27 & 4,59 & 15,24 & 7,54 \\
\hline Student & \multicolumn{2}{|c|}{0.005} & \multicolumn{2}{|c|}{ 8,04E-06 } \\
\hline & \multicolumn{4}{|c|}{ Procédure de suivi des contours } \\
\hline Moyenne/20 & 1,59 & 3,28 & 15,24 & 7,21 \\
\hline Student & \multicolumn{2}{|c|}{0,151} & \multicolumn{2}{|c|}{ 1,18E-06 } \\
\hline & \multicolumn{4}{|c|}{ Procédure des pressions-dépressions } \\
\hline Moyenne/20 & 5,71 & 5,67 & 18,73 & 9,18 \\
\hline Student & \multicolumn{2}{|c|}{0,932} & \multicolumn{2}{|c|}{ 5,18E-10 } \\
\hline & \multicolumn{4}{|c|}{ Procédure de contact statique } \\
\hline Moyenne/20 & 0 & 0 & 4,76 & 0,33 \\
\hline Student & \multicolumn{2}{|c|}{ pas calculable } & \multicolumn{2}{|c|}{0,0002} \\
\hline & \multicolumn{4}{|c|}{ Procédure de soupèsement } \\
\hline Moyenne/20 & 0 & 0 & 3,17 & 0,33 \\
\hline Student & \multicolumn{2}{|c|}{ pas calculable } & \multicolumn{2}{|c|}{0,005} \\
\hline & \multicolumn{4}{|c|}{ Procédure de frottement latéral } \\
\hline Moyenne/20 & 6,03 & 5,90 & 16,19 & 14,43 \\
\hline Student & \multicolumn{2}{|c|}{0,938} & \multicolumn{2}{|c|}{0,250} \\
\hline
\end{tabular}

GE : groupe expérimental

GT : groupe témoin

des étudiants et la perception des éléments anatomiques doit rendre plus performant l'exercice de leur futur métier. La transposition didactique utilise les six procédures palpatoires dans la double fonction d'outil de formation et d'évaluation contrôle.

En ce qui concerne l'aspect formatif, cette stratégie nous amène à penser que l'étudiant a acquis l'habileté gestuelle nécessaire pour toutes les palpations qu'il aura à réaliser. Il a donc amélioré sa compétence professionnelle en combinant des connaissances, du savoir-faire, de l'expérience et un comportement s'exerçant dans un contexte précis ${ }^{[2]}$.

En ce qui concerne l'aspect évaluatif, et bien que la grille d'évaluation proposée soit discutable en termes de traçabilité des réelles perceptions, le formateur peut bénéficier d'un outil d'évaluation-contrôle. Nous pointons à ce niveau l'importance de la valida- tion des compétences au moment du passage d'un cycle d'études à un autre.

Cette étude nous a permis de mettre en évidence l'acquisition de la compétence haptique chez les masseurs-kinésithérapeutes en termes d'évaluation palpatoire. Nous sommes maintenant en droit de nous demander si cette compétence permet l'élaboration d'un traitement de thérapie manuelle plus efficient (mobilisation tissulaire ou massage antalgique). À titre d'exemple, on peut questionner le fait que l'apprentissage palpatoire haptique puisse être transféré en pratique clinique. Si tel était le cas, on peut s'interroger quant à l'utilité d'un tel transfert pour adapter efficacement le massage antalgique à la lutte contre la douleur chronique. Ces interrogations nous amènent à envisager une poursuite de nos investigations dans ce sens. 


\section{Déclaration d'intérêts}

L'auteur ne déclare aucun conflit d'intérêt en lien avec le contenu de cet article.

\section{Approbation éthique}

Non sollicitée

\section{Références}

1. CNOM. Ordre des masseurs-kinésithérapeutes. [Online]. Disponible sur : http://www.ordremk.fr/wpcontent/unploads/2013/03/Le-référentiel.pdf

2. Zarifian P. Objectif compétence. Pour une nouvelle logique. Paris : Liaisons, 1999.

3. De Singly F. L'enquête et ses méthodes. Le questionnaire. 2ème éd. Paris : Armand Colin, 2005.

4. Wittorski R. Professionnalisation et développement professionnel. Paris : L'Harmattan, 2007.

5. Choplin A. L'apprentissage de l'exploration tactile des étudiants masseurs-kinésithérapeutes. Kinésithérapie Scientifique 2012;538:25-30.

6. Revesz G. System der optischen und haptishen Raumtaüschungen.Zeitcrift für Physiologie 1934;131:296375.

7. Gibson J. The senses considered as perceptual systems. Boston: Houghton Mifflin Compagny, 1966,

8. Lederman S. Hands movements : a window into haptic object recognition. Cognitive Psychology 1987;19: 342-68

9. Klatzky RL. Haptic identification of objects and their depiction. Perception and psychophysics 1993;54: 170-78
10. Hatwell Y. Toucher pour connaître. Psychologie cognitive de la perception tactile manuelle. Paris : PUF, 2000.

11. Gentaz E. La main, le cerveau et le toucher. Paris : Dunod, 2009.

12. Chevallard Y. La transposition didactique : Du savoir savant au savoir enseigné. Grenoble : La pensée sauvage, 1985.

13. Winnykamen F. Apprendre en Imitant. Paris : PUF, 1990.

14. Vytgotski L. Pensée et langage. Paris : La Dispute, 1997.

15. Piaget J. La naissance de l'intelligence chez l'enfant. ( $3^{\text {ème }}$ éd.) Neuchâtel : Delachaux et Niestlé, 1959.

16. Wallon H. De l'acte à la pensée. Paris : Flammarion, 1942/1970.

17. Clot Y, Faïta D. Genres et Styles en analyse du travail. Concepts et Méthodes. Travailler 2000;4:7-42.

18. Vial M. Se répérer dans les modèles de l'évaluation : histoire, modèles, outils. Bruxelles : De Boeck, 2012.

19. Landis JR. The Measurement of Observer Agreement for Categorical Data. Biometrics, 1977;33:159-74

20. Oddone I, Re A, Briante G. Redécouvrir l'expérience ouvrière : vers une autre psychologie du travail? Paris: Messidor/Editions Sociales, 1981:28-29

21. Clot Y, Faïta D, Fernandez G, Scheller L. Entretiens en autoconfrontation croisée : une méthode en clinique de l'activité. Pistes 2000;2:1-7

Correspondance et offprints: Arnaud Choplin. Institut de Formation en Masso-Kinésithérapie Niçois, Centre Hospitalier Universitaire de l'Archet, 151 Route Saint Antoine de Ginestière, 06202 Nice Cedex, France.

Mailto : choplin@unice.fr 


\section{Annexe}

Annexe $\mathrm{n}^{\circ} 1$ : Modélisation des six procédures haptiques sur la palpation de la hanche.

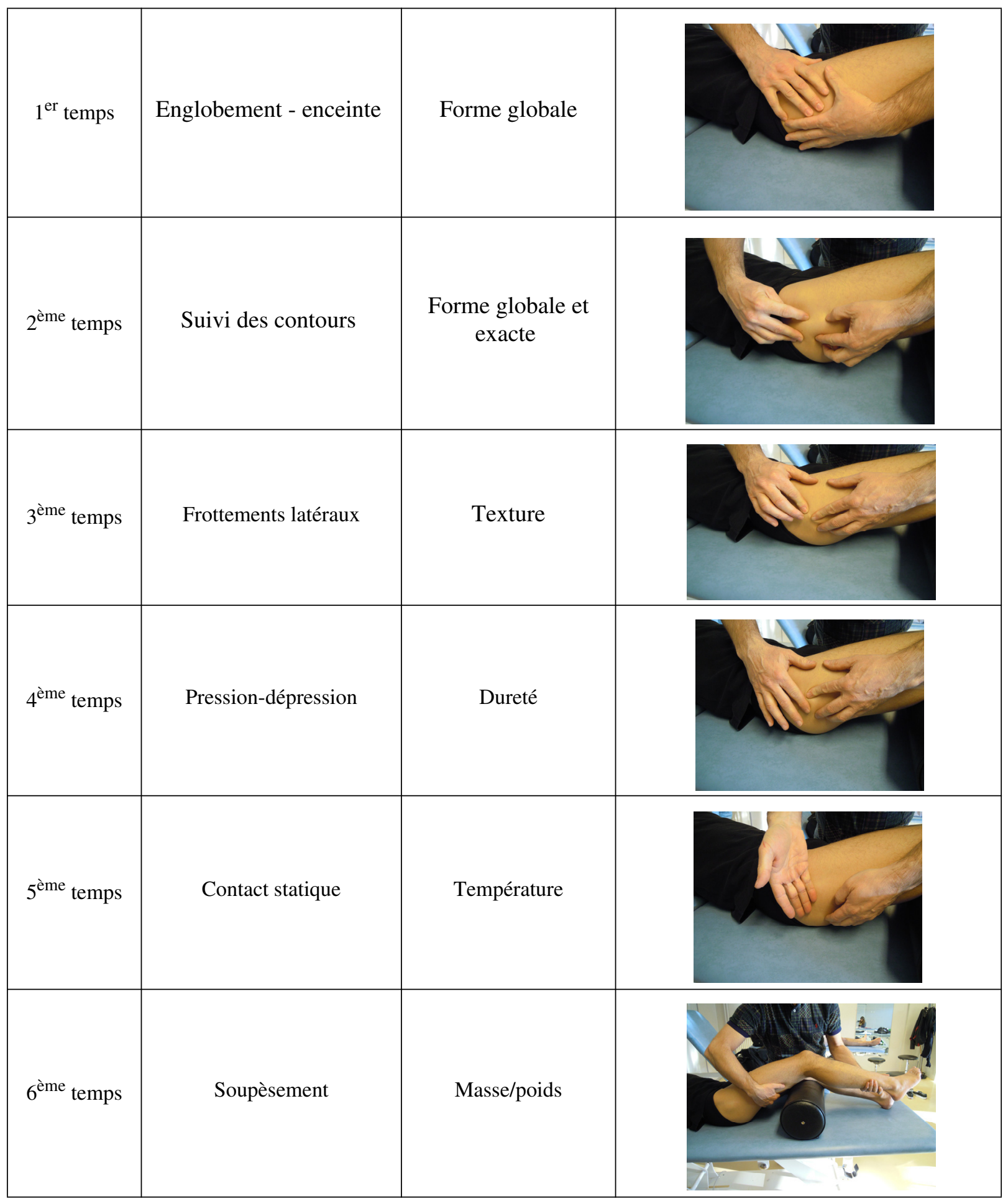


Annexe $\mathrm{n}^{\circ} 2$ : Fiche de recueil de l'information, évaluations initiale et finale (Variables dépendantes : vitesse et qualité).

Recueil d'informations initiales d'octobre

Recueil d'informations finales de février-mars

Nom :

Prénom :

Groupe d'appartenance : 1 ou 2

Date :

Sujet : organiser la palpation thérapeutique de...

PARTIE PRATIQUE :

Installation du patient et réglage de la table

Fiche de recueil de l'information, évaluations initiale et finale

\begin{tabular}{|l|l|l|l|}
\hline Ordre d'apparition & Adapté à & Action pratique & Réalisé \\
\hline Enveloppement & Idée approximative & Avec toute la main dominante & Oui-non \\
\hline Suivi des contours & Forme exacte & Avec le bout des doigts de la main dominante & Oui-non \\
\hline Contact statique & La température & Avec le dos de la main & Oui-non \\
\hline Frottement latéral & $\begin{array}{l}\text { La texture }: \text { rugosité } \\
\text { et aussi la forme }\end{array}$ & $\begin{array}{l}\text { Déplacement latéral dans tous les sens sans } \\
\text { quitter le contact avec la peau }\end{array}$ & Oui-non \\
\hline Pression dépression & La dureté & $\begin{array}{l}\text { Succession d'appui relâchement de l'index } \\
\text { «palpeur » sans quitter le contact avec la } \\
\text { peau }\end{array}$ & Oui-non \\
\hline Soupèsement soulèvement & Au poids & Si possible & Oui-non \\
\hline
\end{tabular}

Précisions pour l'évaluateur :

Les travaux de Lederman et Klatzky (Lederman, 1987, pp. 342-368) permettent d'identifier six principales procédures exploratoires (Gentaz, 1999, pp. 3-4).

1) L'enveloppement permet de donner une idée approximative de l'objet touché

2) Le suivi des contours permet de confirmer la forme globale et d'être sur la forme exacte de l'objet

3) Le contact statique permet une indication de température de l'objet et plus approximativement donne une information sur la forme, la taille, la texture et la dureté

4) Le frottement latéral permet de déterminer la texture de l'objet

5) La pression permet de déterminer la dureté de l'objet

6) Le soupèsement-soulèvement détermine le poids de l'objet.

D'après le tableau de correspondance entre les six principales procédures exploratoires (Lederman, 1987) et la spécificité des corpuscules. 
Annexe $n^{\circ} 3$ : Recueil des données pour le calcul du coefficient de Cohen’s KAPPA.

Nombre de procédures haptiques utilisées par les 12 étudiants de deuxième cycle dans le cadre de l'épreuve de réplicabilité de la grille d'évaluation utilisée pour cette étude

\begin{tabular}{|c|c|c|}
\hline $\begin{array}{c}\text { Kappa / grille } \\
\text { d'évaluation }\end{array}$ & \multicolumn{2}{|c|}{$\begin{array}{c}\text { Nombre de procédures haptiques utilisées } \\
\text { (sur 6) }\end{array}$} \\
\hline 12 étudiants $\mathrm{n}^{\circ}$ & Cadre MK & Master Recherche \\
\hline $\mathrm{a}$ & 4 & 5 \\
\hline $\mathrm{b}$ & 5 & 5 \\
\hline $\mathrm{c}$ & 3 & 3 \\
\hline $\mathrm{d}$ & 2 & 2 \\
\hline $\mathrm{e}$ & 4 & 4 \\
\hline $\mathrm{f}$ & 5 & 5 \\
\hline $\mathrm{g}$ & 3 & 3 \\
\hline $\mathrm{h}$ & 3 & 4 \\
\hline $\mathrm{i}$ & 4 & 4 \\
\hline $\mathrm{j}$ & 6 & 6 \\
\hline $\mathrm{k}$ & 3 & 4 \\
\hline $\mathrm{l}$ & 3 & 3 \\
\hline
\end{tabular}

MK : masseur kinésithérapie

Annexe $n^{\circ} 4$ : Résultats exhaustifs des données brutes.

Résultats de l'utilisation des procédures palpatoires du groupe témoin par année.

\begin{tabular}{|c|c|c|c|c|c|c|c|c|c|c|c|}
\hline \multirow{2}{*}{\multicolumn{2}{|c|}{\begin{tabular}{|l} 
Groupe Témoin \\
61 étudiants
\end{tabular}}} & \multicolumn{3}{|c|}{ Évaluation initiale } & \multicolumn{3}{|c|}{ Évaluation finale } & \multicolumn{3}{|c|}{$\begin{array}{l}\text { Variation des différences } \\
\text { par année }\end{array}$} & \multirow{2}{*}{$\begin{array}{l}\text { Diff. } \\
\text { totale }\end{array}$} \\
\hline & & 2011 & 2012 & 2013 & 2011 & 2012 & 2013 & 2011 & 2012 & 2013 & \\
\hline \multirow{6}{*}{$\begin{array}{l}\text { Les six } \\
\text { procédures } \\
\text { palpatoires }\end{array}$} & enveloppement & 0 & 3 & 11 & 8 & 3 & 12 & 8 & 0 & 1 & 9 \\
\hline & \begin{tabular}{|l} 
suivi des contours \\
\end{tabular} & 5 & 3 & 2 & 13 & 4 & 5 & 8 & 1 & 3 & 12 \\
\hline & contact statique & 0 & 0 & 0 & 0 & 0 & 1 & 0 & 0 & 1 & 1 \\
\hline & frottement latéral & 2 & 2 & 14 & 15 & 15 & 14 & 13 & 13 & 0 & 26 \\
\hline & \begin{tabular}{|l} 
pression-dépression \\
\end{tabular} & 4 & 9 & 4 & 14 & 2 & 12 & 10 & 7 & 8 & 11 \\
\hline & soupèsement & 0 & 0 & 0 & 0 & 0 & 1 & 0 & 0 & 1 & 1 \\
\hline \multicolumn{2}{|l|}{ TOTAL } & 11 & 17 & 31 & 50 & 24 & 45 & 39 & 21 & 14 & \\
\hline
\end{tabular}


Résultats de l'utilisation des procédures palpatoires du groupe expérimental par année.

\begin{tabular}{|c|c|c|c|c|c|c|c|c|c|c|c|}
\hline \multirow{2}{*}{\multicolumn{2}{|c|}{$\begin{array}{l}\text { Groupe Expérimental } \\
63 \text { étudiants }\end{array}$}} & \multicolumn{3}{|c|}{ Évaluation initiale } & \multicolumn{3}{|c|}{ Évaluation finale } & \multicolumn{3}{|c|}{$\begin{array}{l}\text { Variation des différences } \\
\text { par année }\end{array}$} & \multirow{2}{*}{$\begin{array}{l}\text { Diff. } \\
\text { totale }\end{array}$} \\
\hline & & 2011 & 2012 & 2013 & 2011 & 2012 & 2013 & 2011 & 2012 & 2013 & \\
\hline \multirow{6}{*}{$\begin{array}{l}\text { Les six } \\
\text { procédures } \\
\text { palpatoires }\end{array}$} & enveloppement & 1 & 1 & 2 & 18 & 15 & 15 & 17 & 14 & 13 & 44 \\
\hline & suivi des contours & 1 & 1 & 2 & 18 & 15 & 15 & 13 & 15 & 15 & 43 \\
\hline & contact statique & 0 & 0 & 0 & 8 & 5 & 2 & 8 & 5 & 2 & 15 \\
\hline & frottement latéral & 0 & 1 & 18 & 15 & 19 & 17 & 15 & 18 & 1 & 32 \\
\hline & pression-dépression & 5 & 7 & 6 & 21 & 19 & 19 & 16 & 12 & 13 & 41 \\
\hline & soupèsement & 0 & 0 & 0 & 4 & 1 & 5 & 4 & 1 & 5 & 10 \\
\hline \multicolumn{2}{|l|}{ TOTAL } & 7 & 10 & 28 & 84 & 74 & 73 & 73 & 65 & 48 & \\
\hline
\end{tabular}

